\title{
APPLICATION OF WAVELET TRANSFORM TECHNIQUES TO VIBRATION STUDIES
}

\author{
Ülo LEPIK \\ Institute of Applied Mathematics, University of Tartu, Vanemuise 46, 51014 Tartu, Estonia; \\ ylepik@ut.ee \\ Received 24 April 2001 \\ Abstract. Wavelet transform techniques are applied to analysis of linear vibrations. It is shown \\ that in some simple cases wavelet transform can be accomplished analytically. Damped and \\ forced vibrations of single and two degrees of freedom are considered. The achieved results \\ can be used for interpreting more complicated cases.
}

Key words: linear vibrations, damped and forced vibrations, Fourier transform, wavelets.

\section{INTRODUCTION}

The Fourier transform is a valuable tool for analysing time series. It transforms the signal $f=f(t)$ to a frequency diagram $F=F(\omega)$; if this diagram has only sharp peaks, then the motion consists of harmonic components corresponding to the peak frequencies. It should be mentioned that the Fourier transform has also a serious disadvantage. To compute $F=F(\omega)$, we must integrate $x(t)$ over all time and, therefore, the motion cannot be localized in time. To illustrate this circumstance, let us consider the case of intermittent chaos. Here we have chaotic motion which in short time intervals is interchanged with regular motion. The Fourier transform gives us a global overview of the process: it allows us to establish the chaotic character of the motion, but does not give any information about the intervals of regular motion. To sum up: the Fourier transform completely localizes the time series in frequency, but the results are completely delocalized in time.

One way to break out of this deadlock is to use the wavelet transform, which was introduced in the works of J. Morlet in the early 1980s. At the outset the wavelet method was considered as a mathematical curiosity, but due to extensive 
research in the 1990s it has turned to a well-grounded and powerful mathematical tool with many practical applications. Further information about the wavelet technique can be found in the corresponding literature (see, e.g., $\left[{ }^{1,2}\right]$ ).

The wavelet method has been applied in only few contributions in structural mechanics. Here we shall recite the works about linear vibrations. In the textbook by Newland $\left[{ }^{2}\right]$ an example of damped vibrations is presented; the problem is solved with the aid of the Daubechies wavelets. An example of forced singledegree-of-freedom (SDoF) vibration is discussed by Kyprianou and Staszewski $\left[{ }^{3}\right]$. Staszewski $\left[{ }^{4}\right]$ has also analysed damping in SDoF and multi-degree-offreedom systems. Wong and Chen $\left[{ }^{5}\right]$ considered time series consisting of two different harmonic modes. In papers $\left[{ }^{3-5}\right]$ the Morlet wavelet was used. Zheng and McFadden $\left[{ }^{6}\right]$, making use of the Gabor transform considered a signal which is the sum of two complex sinusoids. This method is also applied to analysing vibrations of a gearbox.

In most of these papers linear vibration problems are considered as examples to illustrate theoretical results. The problems were solved by computer making use of the discrete wavelet transform.

In this paper it is shown that in some simple cases the wavelet transform can be carried out analytically. This circumstance, of course, significantly simplifies numerical work. The obtained analytical solutions are also useful in interpreting numerical results achieved for more complicated problems.

\section{THEORETICAL BACKGROUND}

Let us consider a function $f=f(t)$ satisfying the condition

$$
\int_{-\infty}^{+\infty}|f(t)|^{2} d t<\infty
$$

The Fourier transform of $f=f(t)$ and its inverse will be defined as

$$
F(\omega)=\int_{-\infty}^{+\infty} f(t) e^{-i \omega t} d t, \quad f(t)=\frac{1}{2 \pi} \int_{-\infty}^{+\infty} F(\omega) e^{i \omega t} d t .
$$

For analysing the function $f(t)$ we introduce a wavelet

$$
g(t, a, b)=\frac{1}{\sqrt{a}} g\left(\frac{t-b}{a}\right),
$$

where $g=g(t)$ is the mother wavelet, $a>0$ is the dilation parameter, and $b$ is the translation parameter.

The wavelet transform is

$$
W(a, b)=\frac{1}{\sqrt{a}} \int_{-\infty}^{+\infty} f(t) \bar{g}\left(\frac{t-b}{a}\right) d t,
$$

where the symbol $\bar{g}$ denotes the conjugate form of $g$. 
Making use of the Parceval identity

$$
2 \pi \int_{-\infty}^{+\infty} f(t) g(t, a, b) d t=\int_{-\infty}^{+\infty} F(\omega) \bar{G}(\omega, a, b) d \omega
$$

Eq. (4) can be put into the form

$$
W(a, b)=\frac{1}{2 \pi \sqrt{a}} \int_{-\infty}^{+\infty} F(\omega) \bar{G}(\omega, a, b) d \omega .
$$

Here $G(\omega, a, b)$ is the Fourier transform of $g(t, a, b)$.

For evaluating $G(\omega, a, b)$ we start from the formula

$$
G(\omega, a, b)=\int_{-\infty}^{+\infty} g\left(\frac{t-b}{a}\right) e^{-i \omega t} d t .
$$

By substituting $t=a \tau+b$ we find

$$
G(\omega, a, b)=a e^{-i \omega b} \int_{-\infty}^{+\infty} g(\tau) e^{-i a \omega \tau} d \tau=a G(a \omega) e^{-i \omega b}
$$

where $G(\omega)$ is the Fourier transform of the mother wavelet $g(t)$ :

$$
G(\omega)=\int_{-\infty}^{+\infty} g(t) e^{-i \omega t} d t .
$$

Taking into account Eqs. (7) and (8), we get:

$$
W(a, b)=\frac{1}{2 \pi} \sqrt{a} \int_{-\infty}^{+\infty} F(\omega) \bar{G}(a \omega) e^{i \omega b} d \omega .
$$

This result can be interpreted as an inverse Fourier transform of the function $\sqrt{a} F(\omega) \bar{G}(a \omega)$. Equation (10) is presented in many textbooks about wavelet transform (see, e.g., $\left[{ }^{1}\right]$ ).

The function $W(a, b)$ is usually complex:

$$
W=\operatorname{Re}(W)+i \operatorname{Im}(W) .
$$

For interpreting the results we shall introduce the functions $\left[{ }^{5}\right]$

$$
\begin{aligned}
|W(a, b)| & =\left[\operatorname{Re}^{2}(W)+\operatorname{Im}^{2}(W)\right]^{1 / 2}, \\
\phi(a, b) & =\arctan \frac{\operatorname{Im}(W)}{\operatorname{Re}(W)} .
\end{aligned}
$$

In the following we shall fix the dilation parameter $a$ and put together the diagrams $(b,|W|)$ and $(b, \phi)$, where $b$ stands for time. The diagram $(b,|W|)$ 
matches the behaviour of the amplitude of the signal and $(b, \phi)$ shows the phase as a function of time.

We shall consider three wavelets, for which the Fourier transforms can be carried out analytically.

(i) Morlet wavelet. Here the mother wavelet has the form

$$
g(t)=e^{i \omega_{0} t} e^{-0.5 t^{2}} .
$$

Its Fourier transform gives

$$
G(\omega)=\sqrt{2 \pi} \exp \left[-0.5\left(\omega-\omega_{0}\right)^{2}\right] .
$$

Reconstruction of $g$ from $G$ is possible only if the admissibility condition

$$
C=\int_{-\infty}^{+\infty} \frac{|G(\omega)|^{2}}{|\omega|} d \omega<\infty
$$

is fulfilled. Since in the present case $G(0) \neq 0$, it is not possible to satisfy the condition (16) exactly. Therefore, following the papers $\left[^{1,5}\right]$, we shall satisfy Eq. (16) approximately by taking $\omega_{0}=5$.

(ii) Haar wavelet. This wavelet is defined as

$$
g(t)=\left\{\begin{array}{l}
1 \text { for } 0 \leq t<0.5 \\
-1 \text { for } 0.5 \leq t<1 \\
0 \quad \text { elsewhere }
\end{array}\right.
$$

Carrying out the Fourier transform, we find

$$
G(\omega)=-\frac{i}{\omega}\left(1-e^{0.5 i \omega}\right)^{2} .
$$

(iii) Mexican hat. Here we have

$$
g(t)=\frac{2}{\sqrt{3}} \pi^{-1 / 4}\left(1-t^{2}\right) e^{-0.5 t^{2}}
$$

and

$$
G(\omega)=\frac{2}{\sqrt{3}} \pi^{-1 / 4} \omega^{2} e^{-0.5 \omega^{2}} .
$$

It is not difficult to check that the condition (16) is satisfied for Eqs. (18) and (20). 


\section{DAMPED VIBRATIONS OF A SDoF SYSTEM}

In this section we shall consider linear vibrations with SDoF, which are described by the equation

$$
\ddot{x}+p \dot{x}+q x=r \psi(t)
$$

where $p, q, r$ are real constants, the function $\psi(t)$ describes forced vibrations.

By applying the Fourier transform to Eq. (21) and taking into account that

$$
F(\dot{x})=i \omega F(\omega), \quad F(\ddot{x})=-\omega^{2} F(\omega),
$$

we find

$$
F(\omega)\left(-\omega^{2}+i p \omega+q\right)=r \Psi(\omega)
$$

where

$$
\Psi(\omega)=\int_{-\infty}^{+\infty} \psi(t) e^{-i \omega t} d t
$$

In the case of free motions $r=0$. Let us denote the roots of the equation $\omega^{2}-i p \omega-q=0$ by $\omega_{1}$ and $\omega_{2}$. Then we find

$$
\omega_{1}=\alpha i+\beta, \quad \omega_{2}=\alpha i-\beta,
$$

where $\alpha=p / 2, \beta=\sqrt{q-p^{2} / 4}$. We shall consider here only the case of low damping; then $q>p^{2} / 4$ and $\beta$ is a real number.

Equation (22) takes the form $F(\omega)\left(\omega-\omega_{1}\right)\left(\omega-\omega_{2}\right)=0$. Consequently, $F(\omega)=0$ if $\omega \neq \omega_{1}$ or $\omega \neq \omega_{2}$.

This fact can be rewritten in the form

$$
F(\omega)=C_{1} \delta\left(\omega-\omega_{1}\right)+C_{2} \delta\left(\omega-\omega_{2}\right),
$$

where the symbol $\delta$ denotes the delta function. The complex constants $C_{1}, C_{2}$ can be calculated in the following way. By executing the inverse Fourier transform to $F(\omega)$ we get

$f(t)=\frac{1}{2 \pi} \int_{-\infty}^{+\infty}\left[C_{1} \delta\left(\omega-\omega_{1}\right)+C_{2} \delta\left(\omega-\omega_{2}\right)\right] e^{i \omega t} d \omega=\frac{1}{2 \pi}\left(C_{1} e^{i \omega_{1} t}+C_{2} e^{i \omega_{2} t}\right)$.

Let us choose $C_{1}=A-B i, C_{2}=0$, where $A, B$ are real constants. Then

$$
x(t)=\operatorname{Re} f(t)=\frac{1}{2 \pi} e^{-\alpha t}(A \cos \beta t+B \sin \beta t) .
$$

This is the general solution for damped vibrations. The constants $A, B$ can be calculated from the initial conditions. 
Making use of these considerations the function $F(\omega)$ can be put into the form

$$
F(\omega)=(A-i B) \delta\left(\omega-\omega_{1}\right) .
$$

Since

$$
\int_{-\infty}^{\infty} h(x) \delta(x-\xi) d x=h(\xi)
$$

it follows from Eq. (10) that

$$
W(a, b)=\frac{\sqrt{a}}{2 \pi}(A-i B) \bar{G}\left(a \omega_{1}\right) e^{i \omega_{1} b} .
$$

Let us consider first the Morlet wavelet. Taking into account Eq. (15) and the fact that $\bar{\omega}_{1}=-\alpha i+\beta$, we obtain

$$
\begin{aligned}
W(a, b)=\frac{\sqrt{a}}{\sqrt{2 \pi}}(A & -i B) \exp \left[-\frac{1}{2}\left(a \beta-\omega_{0}\right)^{2}+\frac{1}{2} a^{2} b^{2}\right] \\
& \times \exp \left[2 a \alpha\left(\omega_{0}-a \beta\right) i\right] \exp [b(-\alpha+i \beta)] .
\end{aligned}
$$

The modulus of $W$ is

$$
|W(a, b)|=\frac{\sqrt{a}}{\sqrt{2 \pi}} \sqrt{A^{2}+B^{2}} e^{-\alpha b} \exp \left[-\frac{1}{2}\left(a \beta-\omega_{0}\right)^{2}+\frac{1}{2} \alpha^{2} a^{2}\right] .
$$

By calculating the phase $\phi$ from Eq. (13) we find

$$
\tan \phi=\frac{\tan (\beta b+\gamma)-B / A}{1+B / A \tan (\beta b+\gamma)},
$$

where $\gamma=a \alpha\left(\omega_{0}-a \beta\right)$.

It is expedient to introduce a new variable $\mu=\arctan B / A$. Now Eq. (28) can be presented in a simple form

$$
\phi=\beta b+\gamma-\mu \pm k \pi,
$$

where $k=0,1,2, \ldots$ and for $k$ such a value for which $-\pi \leq \phi \leq \pi$ should be taken.

Let us analyse these results. According to Eq. (27) the modulus of $W$ with regard to $b$ is an exponentially decaying function. The maximum of $|W(a, 0)|$ is realized for the dilation parameter

$$
a_{\max }=\frac{1}{2\left(\beta^{2}-\alpha^{2}\right)}\left(\omega_{0} \beta+\sqrt{\omega_{0}^{2} \beta^{2}+2\left(\beta^{2}-\alpha^{2}\right)}\right) .
$$

If $\alpha=0$ (undamped vibrations), the function $|W(a, 0)|$ does not depend upon the translation parameter $b$. 
The diagram $\phi=\phi(b)$ is a "sawtooth" function with a period $\Delta b=2 \pi / \beta$. The slope of the inclined lines in this diagram does not depend upon the parameter $a$.

As an example the case $p=0.05, q=0.15, r=0, A=0, B=1$ is considered. According to Eq. (30) we find $a_{\max }=13.25$. Time history, modulus, and phase of the wavelet function $W$ for this value of the dilation parameter $a$ are shown in Fig. 1.

The solutions to the Haar and Mexican hat wavelets can be found in a similar way. It is easy to see that the diagram $(b, \phi)$ is also a sawtooth function with the period $\Delta b=2 \pi / \beta$. In the $(b,|W|)$-diagram also the multiplier $\exp (-2 b)$, which causes exponential decay, appears. For the values of $|W(a, 0)|$ we get the following results:

(i) Haar wavelet

$$
|W(a, 0)|=\frac{1}{2 \pi \sqrt{a}} \sqrt{A^{2}+B^{2}} \frac{1}{\sqrt{\alpha^{2}+\beta^{2}}}\left(1+e^{-a \alpha}-2 e^{-0.5 a \alpha} \cos 0.5 a \beta\right),
$$

(ii) Mexican hat

$$
|W(a, 0)|=\frac{1}{\sqrt{3}} \frac{a^{5 / 2}}{\pi^{5 / 4}} \sqrt{A^{2}+B^{2}}\left(\beta^{2}-\alpha^{2}\right) \exp \left[-0.5\left(\beta^{2}-\alpha^{2}\right) a^{2}\right] .
$$

Normalized quantities $|W(a, 0)|$ versus the dilation parameter $a$ for the three wavelets considered above are plotted in Fig. 2. It follows from this diagram that the Mexican hat gives the best resolution in frequency domain.
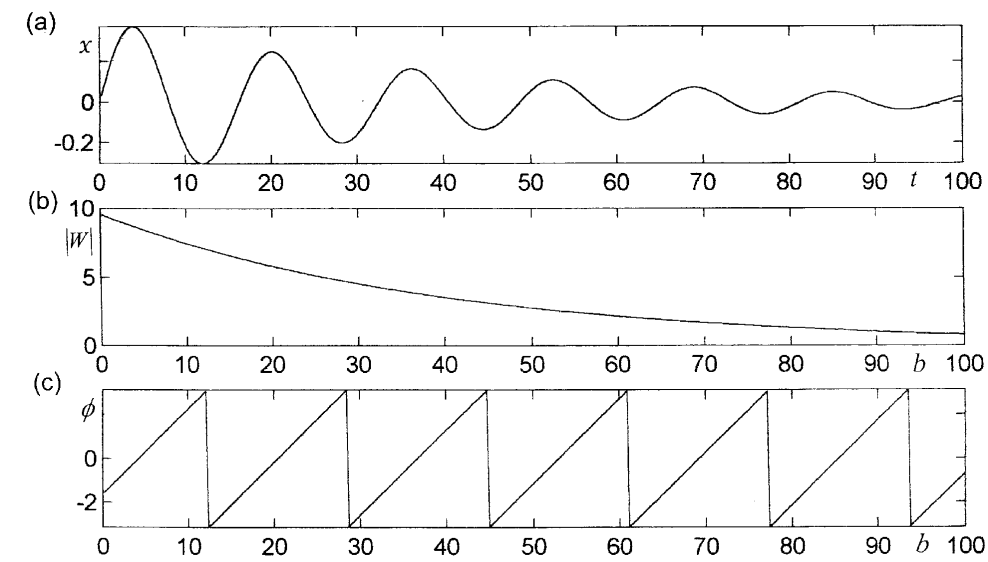

Fig. 1. Damped vibrations for $p=0.05, q=0.15, r=0, a=13.25$. (a) Time history; (b) modulus of the Morlet wavelet $W(a, b)$; (c) phase $\phi(a, b)$. 


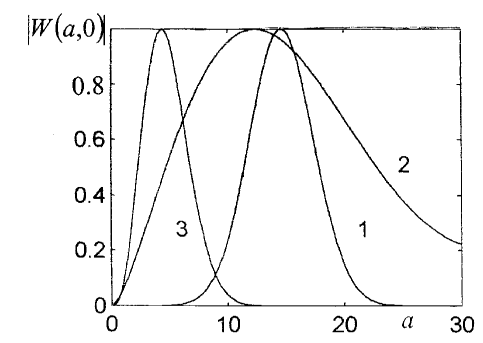

Fig. 2. Normalized modulus of $W(a, 0)$ as a function of the dilation parameter $a$ for (1) Morlet wavelet, (2) Haar wavelet, and (3) Mexican hat.

\section{FORCED SDoF VIBRATION}

For this case $r \neq 0$ in Eq. (21), and we shall seek the solution in the form $x(t)=x_{1}(t)+x_{2}(t)$, where $x_{1}(t)$ is the general solution of the homogeneous equation

$$
\ddot{x}_{1}+p \dot{x}_{1}+q x_{1}=0
$$

and $x_{2}(t)$ is some particular solution of Eq. (21).

The wavelet solution to Eq. (33) was discussed in Section 3. Now we shall turn our attention to Eq. (21). It follows from Eq. (22) that

$$
F(\omega)=\frac{r \Psi(\omega)}{-\omega^{2}+i p \omega+q}
$$

The following analysis is carried out for $\psi(t)=\cos \nu t$. We obtain

$$
F(\omega)=\frac{r}{2} \frac{\delta(\omega-\nu)+\delta(\omega+\nu)}{-\omega^{2}+i p \omega+q} .
$$

The function $F(\omega)$ has at $\omega=\omega_{1}$ and $\omega=\omega_{2}$ indeterminancies in the form $0 / 0$. By applying the Hospital rule and taking into account that

$$
\delta^{\prime}(\omega \pm \nu)=-\frac{\delta(\omega \pm \nu)}{\omega \pm \nu}
$$

we find $F\left(\omega_{1}\right)=F\left(\omega_{2}\right)=0$.

In view of Eq. (34) we can put Eq. (10) into the form

$$
\begin{aligned}
& W(a, b)= \\
& \frac{r \sqrt{a}}{\left(q-\nu^{2}\right)^{2}+p^{2} \nu^{2}}\left\{\left(q-\nu^{2}-i p \nu\right) \bar{G}(a \nu) e^{i \nu b}+\left(q-\nu^{2}+i p \nu\right) \bar{G}(-a \nu) e^{-i \nu b}\right\} .
\end{aligned}
$$


First we shall consider the Morlet wavelet. According to Eq. (15) we have

$$
G( \pm a \nu)=\sqrt{2 \pi} \exp \left[-0.5\left(a \nu \mp \omega_{0}\right)^{2}\right] .
$$

Since $a>0, \nu>0, \omega_{0}=5$, we have $G(-a \nu) \approx 0$ and the second term in Eq. (35) can be neglected. By calculating the modulus $|W|$ and phase $\phi$ from Eq. (35) we find

$$
\begin{aligned}
|W(a, b)| & =r \sqrt{\frac{\pi a}{2}} \exp \left[-0.5\left(a \nu-\omega_{0}\right)^{2}\right], \\
\tan \phi & =\frac{\tan \nu b-s}{s \tan \nu b+1}, \quad s=\frac{p \nu}{q-\nu^{2}} .
\end{aligned}
$$

By introducing a new variable $\mu=\arctan s$ we can put Eq. (37) into the form

$$
\phi=\nu b-\mu \pm k \pi \text {. }
$$

So we see that $|W(a, b)|$ does not depend upon $b$ and its maximal value is realized at

$$
a_{\max }=\frac{1}{2 \nu}\left(\omega_{0}+\sqrt{\omega_{0}^{2}+2}\right) .
$$

The $(b, \phi)$-diagram has again the sawtooth form with a period $\Delta b=2 \pi / \nu$.

Solutions on the basis of the Haar and Mexican hat wavelets can be found in a similar way. To be short, we shall present here only the final results. In both cases the function $W(a, b)$ is real and consequently $\phi=0$. Besides, we get for the Haar wavelet

$W(a, b)=$

$\frac{r}{2 \pi \nu \sqrt{a}} \frac{1}{\left(q-\nu^{2}\right)^{2}+p^{2} \nu^{2}}\left[E\left(1-2 \cos \frac{a \nu}{2}+\cos a \nu\right)+F\left(2 \sin \frac{a \nu}{2}-\sin a \nu\right)\right]$,

where

$$
\begin{aligned}
& E=\left(q-\nu^{2}\right) \sin \nu b-p \nu \cos \nu b, \\
& F=-\left(q-\nu^{2}\right) \cos \nu b-p \nu \sin \nu b .
\end{aligned}
$$

For the Mexican hat we obtain

$W(a, b)=\frac{r a^{5 / 2} \pi^{-5 / 4}}{\sqrt{3}} \frac{\nu^{2}}{\left(q-\nu^{2}\right)^{2}+p^{2} \nu^{2}} \exp \left(-0.5 a^{2} \nu^{2}\right)\left[\left(q-\nu^{2}\right) \cos \nu b+\sin \nu b\right]$. 
Now let us turn back to Eq. (21). Its general solution has the form $x(t)=$ $x_{1}(t)+x_{2}(t)$. The Fourier transform gives

$$
F(\omega)=F_{1}(\omega)+F_{2}(\omega)
$$

where $F_{1}(\omega)$ and $F_{2}(\omega)$ are calculated according to Eqs. (24) and (34). The wavelet function is

$$
\begin{aligned}
W(a, b) & =W_{1}(a, b)+W_{2}(a, b) \\
& =\frac{\sqrt{a}}{2 \pi} \int_{-\infty}^{+\infty}\left[F_{1}(\omega)+F_{2}(\omega)\right] \bar{G}(a \omega) e^{i \omega b} d \omega .
\end{aligned}
$$

Next we have to separate the real and imaginary parts in Eq. (42) and evaluate $|W(a, b)|, \phi(a, b)$. Since this procedure demands long and troublesome calculations, it would better be performed by the computer.

Let us consider the following two examples.

Example 1. Here we shall superpose the results obtained in Sections 3 and 4 for $p=0.05, q=0.15, r=1, \nu=2, a=13.25$. The outcome for the Morlet wavelet is plotted in Fig. 3 (here and later on for $a$ a value for which $|W(a, b)|$ is close to $\max _{a, b}|W(a, b)|$ is chosen). It can be seen from the time history diagram that the motion is pulsatory. Since damped free vibrations dominate, the modulus and phase diagrams correspond to this motion. The sawtooth diagram has the period $\Delta b=2 \pi / \beta=16.26$.

(a)

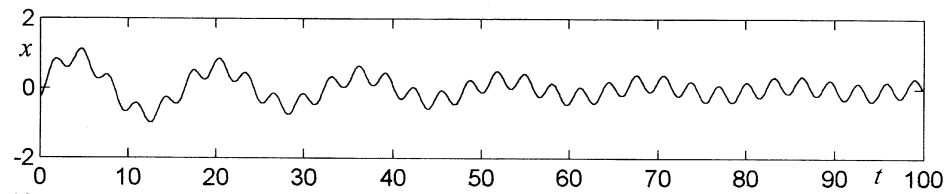

(b)

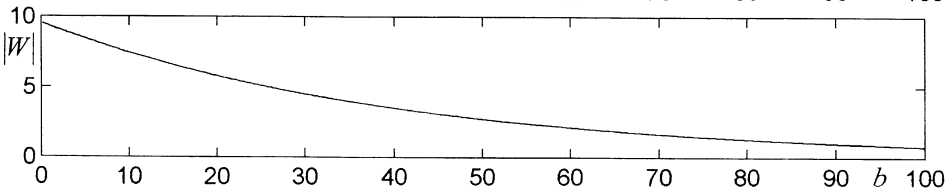

(c)

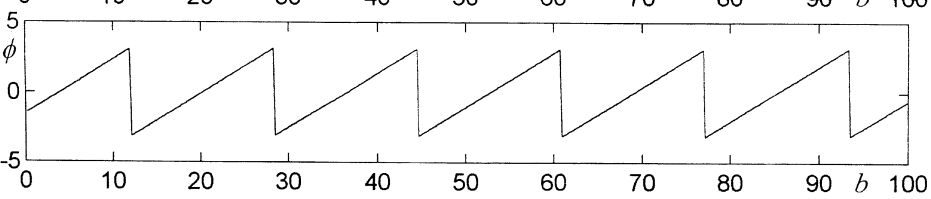

Fig. 3. Forced vibrations for $p=0.05, q=0.15, r=1, \nu=2, a=13.25$. (a) Time history; (b) modulus of the Morlet wavelet $W(a, b)$; (c) phase $\phi(a, b)$. 
Example 2. Let us take $p=q=r=0.1, \nu=0.5, a=14, A=0$, $B=1$ (Fig. 4). The time history diagram shows the well-known fact that at the beginning of the motion free vibrations dominate, but by increasing the time these are overpowered by forced vibrations. This circumstance follows also from the plots in Fig. 4b,c which correspond to the Morlet wavelet. For $b<50$ damped vibrations dominate (this follows from the decay of the modulus $|W|$ and from the period of the sawtooth diagram $\Delta b=2 \pi / \beta=20.1$ ). For forced vibrations there must be $|W(b)|=$ const and the phase diagram has for $b \geq 60$ the period $\Delta b=2 \pi / \nu=12.6$. In the transition zone $b \approx 55-60$ the phase diagram has an irregularity.

\section{FREE VIBRATIONS OF A 2DoF SYSTEM}

In this section we shall consider a two-degree-of-freedom (2DoF) system. For simplicity we shall neglect damping and take the equations of motion in the form

$$
\ddot{x}_{1}+q_{1} x_{1}+q_{2} x_{2}=0, \quad \ddot{x}_{2}+r_{1} x_{1}+r_{2} x_{2}=0,
$$

where $q_{1}, q_{2}, r_{1}, r_{2}$ are real constants.

By applying the Fourier transform to Eqs. (43) we get

$$
\begin{aligned}
& \left(-\omega^{2}+q_{1}\right) F_{1}+q_{2} F_{2}=0, \\
& r_{1} F_{1}+\left(-\omega^{2}+r_{2}\right) F_{2}=0 .
\end{aligned}
$$

(a)

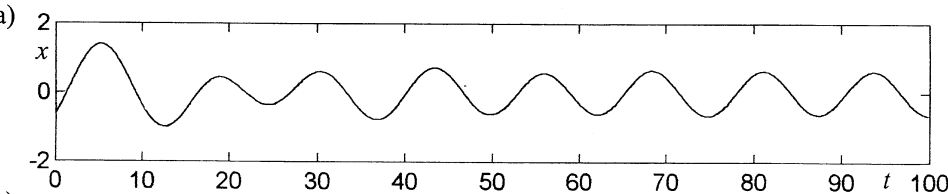

(b)

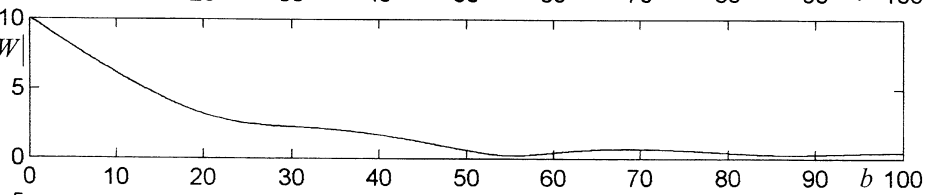

(c)

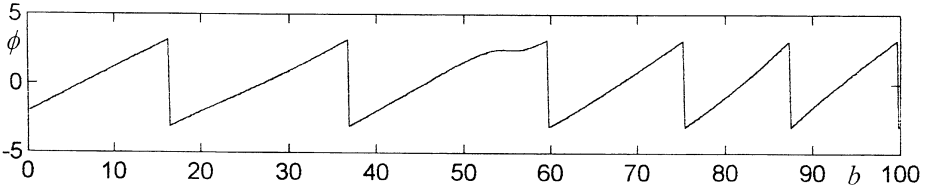

Fig. 4. Forced vibrations for $p=q=r=0.1, \nu=0.5, a=14$. (a) Time history; (b) modulus of the Morlet wavelet $W(a, b)$; (c) phase $\phi(a, b)$. 
This system has a nontrivial solution only if its determinant is zero:

$$
\left(-\omega^{2}+q_{1}\right)\left(-\omega^{2}+r_{2}\right)-r_{1} q_{2}=0 .
$$

We shall confine ourselves to the case $q_{1} / r_{1}>q_{2} / r_{2}$. Then all solutions of Eq. (45) are real and have the form

$$
\omega_{1}=\mu, \quad \omega_{2}=-\mu, \quad \omega_{3}=\nu, \quad \omega_{4}=-\nu,
$$

where

$$
\left\{\begin{array}{l}
\mu \\
\nu
\end{array}=\left[\frac{1}{2}\left(q_{1}+r_{1}\right) \pm \frac{1}{2} \sqrt{\left(q_{1}+r_{2}\right)^{2}-q_{1} r_{2}+r_{1} q_{2}}\right]^{1 / 2} .\right.
$$

The circumstance that the system (44) has a nontrivial solution only for $\omega=\omega_{i}$ $(i=1,2,3,4)$ can be expressed in the form

$$
F_{1}=\sum_{j=1}^{4} A_{j} \delta\left(\omega-\omega_{j}\right), \quad F_{2}=\sum_{j=1}^{4} B_{j} \delta\left(\omega-\omega_{j}\right) \quad(j=1,2,3,4),
$$

where

$$
B_{j}=\frac{\omega_{j}^{2}-q_{1}}{q_{2}} A_{j} .
$$

The constants $A_{j}, B_{j}$ can be evaluated from the initial conditions of the system (43).

The wavelet function for $x_{1}(t)$ is

$$
W(a, b)=\sqrt{2 \pi a} \sum_{j=1}^{4} A_{j} G\left(a \omega_{j}\right) e^{i \omega_{j} b} .
$$

As a numerical example calculations were carried out for $\mu=0.50, \nu=0.45$, $A_{1}=A_{3}=1, A_{2}=A_{4}=-0.5$. The wavelet function was evaluated according to Eq. (49). For calculating the modulus $|W(a, b)|$ and phase $\phi(a, b)$ computer programs were used. The results are plotted in Figs. 5 and 6.

It follows from Fig. 5a that the motion is pulsating. The modulus and phase diagrams are quite near to each other in the case of the Haar and Mexican hat wavelets, but different for the Morlet wavelet. The $(b,|W|)$ diagrams in Fig. 6 practically repeat the pulsating character of the time history diagram, but for the Morlet wavelet the solution of $|W|$ has no high-frequency components (Fig. 5b). The phase diagram for the Morlet wavelet is again a regular sawtooth curve (as in the case of SDoF vibrations), but in the phase diagrams for the Haar and Mexican hat wavelets the inclined segments are crooked. It follows from Figs. 5 and 6 that irregularities of the phase diagram appear for the values of $b$ for which $|W(a, b)| \approx 0$.

A complete analysis of $2 \mathrm{DoF}$ vibrations is difficult since the solution depends upon six parameters: $\mu, \nu, A_{1}, A_{2}, A_{3}, A_{4}$. Nevertheless, the example presented above indicates that interpretation of the wavelet solutions is much more complicated than in the case of SDoF vibrations. 
(a)

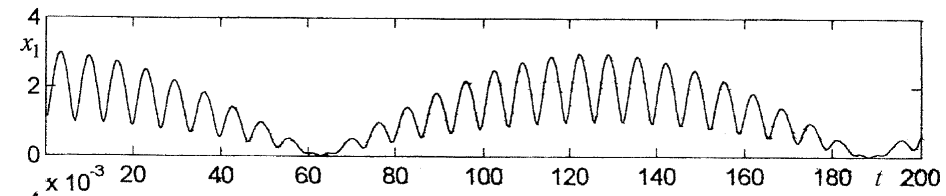

(b)

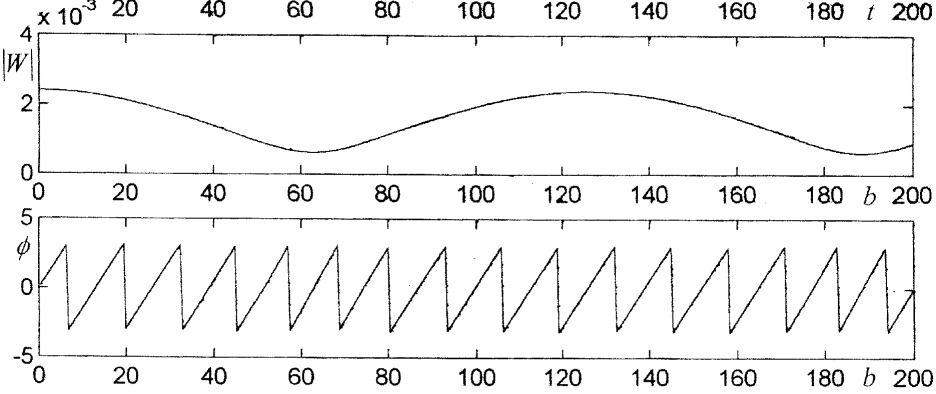

Fig. 5. $2 \mathrm{DoF}$ vibrations for $\mu=0.50, \nu=0.45, A_{1}=A_{3}=1, A_{2}=A_{4}=-0.5, a=3$. (a) Time history for $x_{1}(t)$; (b) modulus of the Morlet wavelet $W(a, b)$; (c) phase $\phi(a, b)$.

(a)

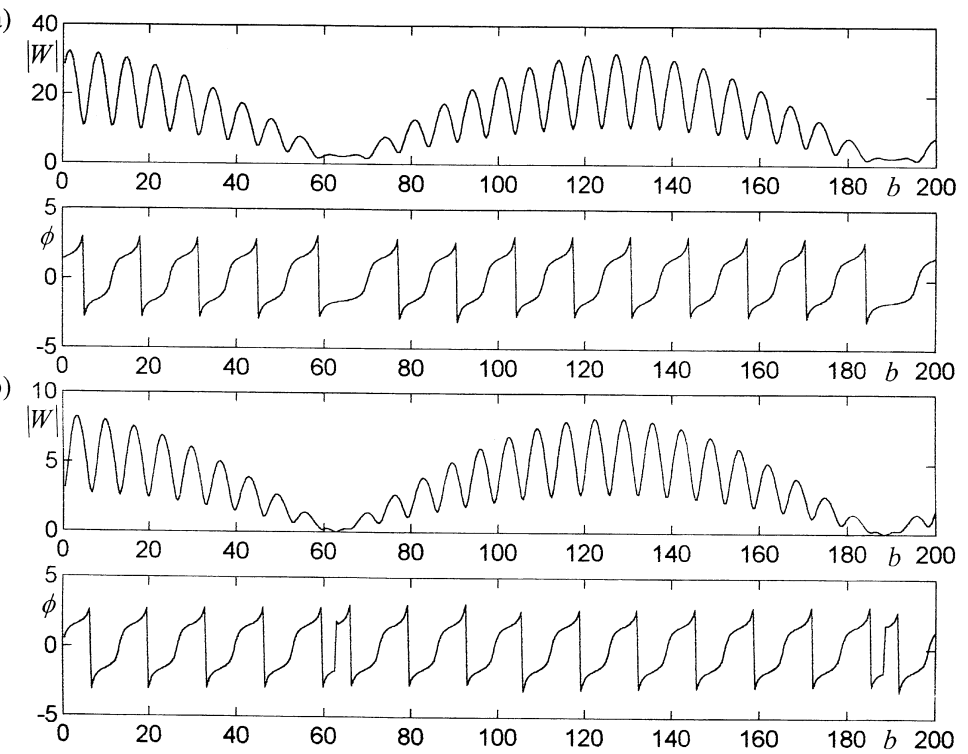

Fig. 6. Modulus $W(a, b)$ and phase $\phi(a, b)$ for the case shown in Fig. 5. (a) Haar wavelet for $a=0.5$; (b) Mexican hat for $a=3$.

\section{CONCLUSIONS}

Linear vibrations were investigated with the aid of the Morlet, Haar, and Mexican hat wavelet transforms. In the case of SDoF vibrations the outcome of 
all these three wavelet transforms was qualitatively the same, but for 2DoF motions the Haar and Mexican hat transforms gave the results essentially differing from those obtained by the Morlet wavelet.

In the case of SDoF motions the diagram $(b,|W|)$ is either a horizontal line (undamped motion) or a curve with exponential decay (damped motion). In the case of the forced motion transition from damped vibrations to forced vibrations can be observed in the modulus and phase diagrams. For 2DoF diagrams, interpretation of the results obtained by the wavelet transforms is much more difficult.

\section{ACKNOWLEDGEMENTS}

The author would like to express his gratitude to Jüri Engelbrecht for reading the manuscript and for his valuable comments. Financial support of the Estonian Science Foundation (grant No. 3380) is gratefully acknowledged.

\section{REFERENCES}

1. Chui, C. K. An Introduction to Wavelets. Academic Pr., San Diego, 1997.

2. Newland, D. E. An Introduction to Random Vibrations, Spectral and Wavelet Analysis. Longman, New York, 1993.

3. Kyprianou, A. and Staszewski, W. J. On the cross wavelet analysis of Duffing oscillator. J. Sound Vibration, 1999, 228, 199-210.

4. Staszewski, W. J. Identification of damping in MDOF systems using the time-scale decomposition. J. Sound Vibration, 1997, 203, 283-305.

5. Wong, L. A. and Chen, J. C. Nonlinear and chaotic behavior of structural system investigated by wavelet transform techniques. Int. J. Non-Linear Mech., 2001, 36, 221-235.

6. Zheng, G. T. and McFadden, P. D. A time-frequency distribution for analysing signals with transient components and its application to vibration analysis. J. Vibr. Acoustics, 1999, 121, 328-333.

\section{LAINIKUTE KASUTAMINE VÕNKUMISTE UURIMISEL}

\section{Ülo LEPIK}

Ühe- ja kahedimensionaalsete võnkumiste uurimisel on rakendatud kolme tüüpi lainikuid. Püstitatud probleem on lineaarsel juhul lahendatav analüütiliselt. Töö tulemusi saab kasutada keerulisemate juhtude analüüsil ja interpreteerimisel. 